\title{
Effect of rapid weight loss and glutamine supplementation on immunosuppression of combat athletes: a double-blind, placebo-controlled study
}

\author{
Aline C. C. Tritto', Mariane T. Amano², Maria E. De Cillo' ${ }^{1}$ Vinicius A. Oliveira², Sandro H. Mendes', Caroline Yoshioka', \\ Hamilton Roschel', Niels Olsen S. Camara², Bruno Gualano', Guilherme G. Artioli',** \\ ${ }^{1}$ Applied Physiology in Nutrition \& Exercise Research Group, University of Sao Paulo, Sao Paulo, Brazil \\ ¿2aboratory of Transplantation Immunobiology, Institute of Biomedical Sciences, University of Sao Paulo, Sao Paulo, Brazil
}

The role of plasma glutamine concentration and glutamine supplementation on immunosuppression was investigated in combat athletes. Twenty-three male athletes were randomly assigned to receive glutamine (21 g/day, $n=12$ ) or placebo (ovalbumin, $n=11)$ for 10 days. Six athletes who did not lose weight served as controls. Athletes were assessed 21 days before $(-21 d), 1$ day before $(-1 d)$ and 5 days after $(+5 d)$ a competition. Weight reduction was similar between glutamine $(-8.2 \% \pm$ $4.1 \%)$ and placebo $(-8.5 \% \pm 2.4 \%)$ and negligible in control $(-0.6 \% \pm 1.4 \%)$. In both weight-loss groups, the majority of athletes reported symptoms of upper respiratory symptoms, as assessed by the Wisconsin upper respiratory symptom survey questionnaire. Only two athletes reported symptoms in the control group. Immune cell function remained unchanged throughout the study except for an increase in neutrophil phagocytic activity (placebo: $-21 d=5,251 \pm 2,986 ;-1 d=17,428 \pm 22,374$;
$+5 d=21,125 \pm 21,934$; glutamine: $-21 d=6,096 \pm 3,549 ;-1 d=11,029 \pm 17,113$; $+5 \mathrm{~d}=28,186 \pm 21,032 \mathrm{FI})$ and a minor change in monocyte phagocytic activity (placebo: $-21 d=4,421 \pm 3,634 ;-1 d=3,329 \pm 6,283 ;+5 d=3,243 \pm$ 2,553; glutamine: $-21 \mathrm{~d}=4,051 \pm 3,186 ;-1 \mathrm{~d}=3,106 \pm 2,625 ;+5 \mathrm{~d}=4,981 \pm$ $4,598)$ in both glutamine and placebo after weight loss. Plasma glutamine and cortisol remained unchanged across the study. creatine kinase levels were increased in placebo $(-21 d=125.2 \pm 54.1 ;-1 d=187.2 \pm$ $73.5 ;+5 \mathrm{~d}=111.3 \pm 59.1 \mathrm{U} / \mathrm{L})$ but not in glutamine $(-21 \mathrm{~d}=136.2 \pm 58.2 ;-1 \mathrm{~d}=$ $168.8 \pm 65.0 ;+5 d=129.7 \pm 64.0 \mathrm{U} / \mathrm{L})$. Rapid weight loss increased the frequency and severity of infection symptoms, but this was neither associated with plasma glutamine depletion nor counteracted by glutamine supplementation.

Keywords: Immune function, Martial arts, Opportunistic infections

\section{INTRODUCTION}

Although combat sport competitions are divided into weight classes, most athletes reduce significant amounts of body weight in the days prior to weigh-in to compete against smaller opponents and thus obtain competitive advantage (Artioli et al., 2016). Achieving large weight reductions in short periods of time requires a combination of different procedures, including severe food restriction and increased training loads (Artioli et al., 2010). Among several adverse effects of rapid weight loss, we highlight the immune system depression which is typically accompanied by increased susceptibility to opportunistic infections (Imai et al.,
2002; Kowatari et al., 2001). Immunosuppression and consequential infections hold potential to impair competitive performance. Anecdotal reports have shown increased frequencies of the common cold, upper respiratory infections, herpes and conjunctivitis in combat athletes during weight loss periods (Imai et al., 2002).

Plasma glutamine levels have long been considered crucial for immune function since the glutamine supply for leukocytes relies entirely on plasma availability (Ardawi and Newsholme, 1983). In neutrophils and macrophages, glutamine is important for rapid energy provision and for the synthesis of membrane lipids and reducing agents (nicotinamide adenine dinucleotide phosphate and
${ }^{*}$ Corresponding author: Guilherme G. Artioli

(iD https://orcid.org/0000-0001-8463-2213

Applied Physiology in Nutrition \& Exercise Research Group, University of Sao Paulo, Av. Prof. Mello Moraes, 65. Sao Paulo, SP 05508-030. Brazil

Tel: +55-11-3091-3077, Fax: +55-11-3091-3077, E-mail: artioli@usp.br

Received: November 7, 2017 / Accepted: January 27, 2018
This is an Open Access article distributed under the terms of the Creative Commons Attribution Non-Commercial License (http://creativecommons.org/licenses/by-nc/4.0/) which permits unrestricted non-commercial use, distribution, and reproduction in any medium, provided the original work is properly cited. 
nicotinamide adenine dinucleotide phosphate, both important for the regulation of oxidative burst) (Curi et al., 1999). In rats, glutamine supplementation improves neutrophil function (Lagranha et al., 2005; Lagranha et al., 2008). In humans, studies with critical and surgical patients (Novak et al., 2002) and healthy exercising volunteers (Castell et al., 1996; Cury-Boaventura et al., 2008; Sasaki et al., 2013) also provide support for a role of plasma glutamine on immune function. In athletes, Kingsbury et al. (1998) observed lower plasma glutamine in those with overtraining syndrome in comparison to their counterparts without chronic fatigue; all athletes presenting clinical symptoms of infection also had glutamine levels below $450 \mathrm{mmol} / \mathrm{L}$. In a placebo-controlled trial, Castell et al. (1996) showed that glutamine supplementation was related to a significant decrease in the incidence of upper respiratory infection symptoms (Castell et al., 1996; Kingsbury et al., 1998). In judoists, glutamine supplementation improved neutrophil function following a single training session by increasing oxidative burst (Sasaki et al., 2013).

However, the efficacy of glutamine supplementation in athletes remains controversial, as some studies have failed to show positive effects on exercise-related immunosuppression (Krzywkowski et al., 2001a, 2001b; Walsh et al., 2000). It is proposed that the discrepancies in the literature may be explained by the degree of plasma glutamine depletion. A marked decrease in plasma glutamine (i.e., values below $400 \mathrm{mmol} / \mathrm{L}$ ) seems to be the threshold beyond which immune dysfunction occurs (Gleeson, 2008). In such cases, glutamine supplementation might be beneficial to the immune system. In contrast, when plasma glutamine is only slightly reduced, which appears to be the case in many studies showing no effect of glutamine supplementation (Krzywkowski et al., 2001a, 2001b; Walsh et al., 2000), immunosuppression would not be linked to plasma glutamine depletion and, therefore, supplementation would not be beneficial.

Rapid weight loss-induced immunosuppression could be related to severe glutamine depletion, since prolonged fasting has been shown to reduce the de novo synthesis of glutamine and increase its degradation via conversion to glucose (i.e., gluconeogenesis [Hankard et al., 1997]). Thus, severe food restriction may deplete circulating glutamine levels (Blanchard et al., 2001). Additionally, intense exercise and increased training loads may reduce plasma glutamine concentrations (Keast et al., 1995; Parry-Billings et al., 1990). Therefore, we sought to examine whether rapid weight loss would deplete plasma glutamine in combat athletes. We also examined whether rapid weight loss would lead to immunosuppression and symptoms of opportunistic infections, and whether glutamine supplementation could ameliorate these responses.

\section{MATERIALS AND METHODS}

\section{Participants}

Fifty-eight male combat athletes volunteered to participate in the study. After being assessed for eligibility, 28 athletes were randomised and allocated to receive either glutamine or placebo; 23 athletes completed the study (glutamine: $\mathrm{n}=12$; age, $20 \pm 2$ years; height, $1.70 \pm 0.07 \mathrm{~m}$; body mass, $71.7 \pm 7.6 \mathrm{~kg}$; body fat, $9.4 \% \pm$ $2.8 \%$ and placebo: $\mathrm{n}=11$; age, $23 \pm 5$ years; height, $1.71 \pm 0.09 \mathrm{~m}$; body mass, $77.4 \pm 13.6 \mathrm{~kg}$; body fat, $12.1 \% \pm 6.1 \%$; Fig. 1). All athletes were competing in official judo $(\mathrm{n}=20)$, wrestling $(\mathrm{n}=1)$, and taekwondo $(\mathrm{n}=2)$ competitions. Inclusion criteria were: (a) 18-30 years of age, (b) minimum training volume of $10 \mathrm{hr} / \mathrm{wk}$, (c) minimum 6 years of experience in the sport, and (d) regularly reduce body weight by $5 \%-10 \%$ before competing. Exclusion criteria were: (a) use of tobacco, (b) use of medications which affect the immune system in the past 2 months, (c) presence of diagnosed infectious disease in the past two months, (d) presence of symptoms suggesting infectious diseases, and (e) use of any nutritional supplement containing glutamine or other substances that may modify immune function. In addition to the 28 athletes who were randomised, eight judo athletes who did not meet a specific inclusion criterion (i.e., not losing weight to compete) were included in the control group in order to control for the effect of competition on the assessed variables (age, $24 \pm 6$ years; height, $1.74 \pm 0.05 \mathrm{~m}$; body mass, $84.4 \pm 24.2 \mathrm{~kg}$; body fat, $15.6 \% \pm 9.8 \%$ ). The study was approved by the Institutional ethics committee (approval number: 2012/11) and all athletes provided written informed consent prior to participation.

\section{Study design}

In this double blind, randomised, placebo-controlled study, athletes were assessed on three separate occasions before a competition: 21 days before (-21d; baseline, a period in which athletes are at their normal body weight), 1 day before (-1d; precompetition, a period in which athletes are close to their competitive weight), and 5 days after a competition $(+5 \mathrm{~d}$; postcompetition, a period in which normal weight is recovered). Those who had to lose weight were randomly allocated into glutamine or isonitrogenated placebo groups. Athletes in the control group were not randomised as they did not receive any treatment and did not lose weight to compete. On all three occasions, assessments were conducted early in the morning and included body mass, body com- 


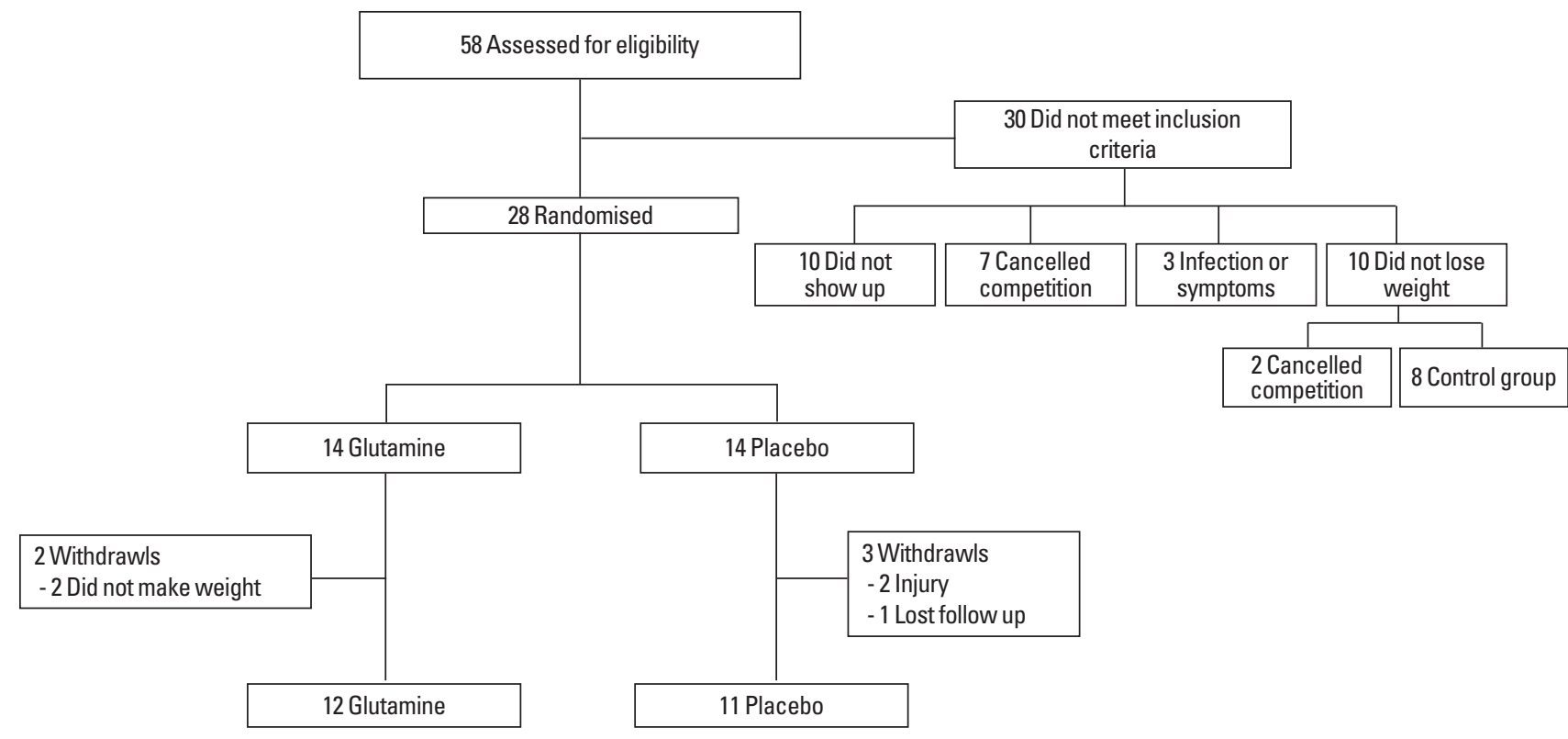

Fig. 1. Flow diagram of randomization and participant allocation to the experimental and control groups.

position, food intake, and symptoms of infection. Fasting morning-time venous blood samples were also collected for the determination of cortisol, total protein, glutamine, creatine kinase (CK) and selected parameters of immune function. In the control group, however, parameters of immune function were not determined due to a malfunction in the equipment resulting in the loss of seven out of the eight samples.

\section{Supplementation protocol}

Athletes received glutamine (Ajinomoto Co. Inc., Tokyo, Japan; $21 \mathrm{~g} /$ day split into 3 daily doses of $7 \mathrm{~g}$ each) or placebo (ovalbumin, Manchester Inc., Sao Paulo, Brazil; 26 g/day split into 3 daily doses of $\sim 8.7 \mathrm{~g}$ each). Ovalbumin was chosen due to its relatively high nitrogen and low glutamine content; the dose used contained the same amount of nitrogen with only $1.9 \mathrm{~g}$ of glutamine. The supplementation period was 10 days, starting on the 5 th day before competition and ending on the 5th day after competition. This supplementation period was chosen because it is well documented that the vast majority of athletes start losing weight in the week of competition, with most weight being reduced in the last 3 days prior to weigh-in (Artioli et al., 2010). In addition, reports indicate that immunosuppression peaks a few days following rapid weight loss (Imai et al., 2002; Kowatari et al., 2001). All supplements were provided in gelatin capsules. At the end of the supplementation period, athletes reported any perceived side effects related to supplementation, returned leftover capsules and attempted to identify which substance they were taking, so that we could confirm blinding efficacy. All methods used for reducing weight were recorded.

\section{Blood sampling}

After collection, 8-hr-fasting venous blood samples were placed on ice and then centrifuged at 2,000 $\mathrm{g}$ for $5 \mathrm{~min}$ at $4{ }^{\circ} \mathrm{C}$. Ethylenediaminetetraacetic acid (EDTA)-plasma was used for the determination of glutamine and $\mathrm{CK}$ whereas serum was used for cortisol and total protein determination. Fresh blood samples collected in EDTA-containing tubes were used for the assessment of neutrophil and monocyte phagocytic activity and oxidative burst.

\section{Serum and plasma analysis}

Plasma CK and plasma glutamine were determined using enzymatic colorimetric-based kits (BT1100200, Biotecnica, Varginha, Brazil; EnzyChromTM, Bioassay Systems, Hayward, CA, USA). Total protein was assessed using the Bradford reagent (Quick Start, BIO-RAD Laboratories, Hercules, CA, USA). Serum cortisol was determined by an enzyme-linked immunosorbent assay (GWBCE6F97, Genway, San Diego, CA, USA).

\section{Body composition}

Skinfold thicknesses were measured from 3 sites (triceps, subscapular, and abdominal). Body fat was estimated according to Lohman's equation, which was previously validated for combat 
athletes (Housh et al., 1989).

\section{Dietary assessment}

Food records were used to assed food intake. For the preweight loss period (i.e., -21d), values from the 3 nonconsecutive days were averaged. The remaining food intake records (i.e., $-3 \mathrm{~d},-2 \mathrm{~d}$, $-1 \mathrm{~d},+5 \mathrm{~d}$ ) were included individually in the analysis. Total energy and macronutrient intake were calculated with the aid of nutritional assessment software (Dietpro, Viçosa, Brazil).

\section{Upper respiratory infection symptoms}

Occurrence and severity of symptoms of the upper respiratory tract were assessed using the Portuguese version of the Wisconsin upper respiratory symptom survey questionnaire (WURSS).

\section{Neutrophil and monocyte phagocytic activity and oxidative burst}

To assess neutrophil and monocyte phagocytic activity, $100 \mu \mathrm{L}$ of fresh blood was incubated for $35 \mathrm{~min}$ at $37^{\circ} \mathrm{C}$ with $25 \mu \mathrm{L}$ of zymosan-FITC $(5 \mathrm{mg} / \mathrm{mL})$. After incubation, $2 \mathrm{~mL}$ of lysis buffer

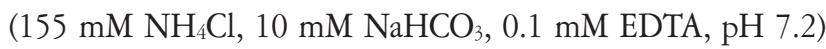
was added; samples were then centrifuged and washed with 2-mL phosphate buffered saline - PBS $\left(\mathrm{KH}_{2} \mathrm{PO}_{4} 5 \mathrm{mM}, \mathrm{K}_{2} \mathrm{HPO}_{4} 1.2\right.$ $\mathrm{mM}, \mathrm{NaCl} 150 \mathrm{mM}, \mathrm{pH}$ 7.0). Fluorescence was read on a flow cytometer (FACS CANTO II, BD Biosciences, San Jose, CA, USA) and data were analysed using the FlowJo software (FlowJo, LLC, Ashland, OR, USA) with monocyte and neutrophil populations being identified by size and granularity.

To assess oxidative burst, $100 \mu \mathrm{L}$ of fresh blood was incubated for $10 \mathrm{~min}$ at room temperature with $2 \mathrm{~mL}$ of lysis buffer and then $3 \mathrm{~mL}$ of PBS-serum (phosphate-buffered saline solution plus $2 \%$ bovine serum) was added. Samples were then centrifuged ( 400 $\mathrm{g}, 4^{\circ} \mathrm{C}, 5 \mathrm{~min}$ ) and cells resuspended in $1 \mathrm{~mL}$ of PBS-serum. Cells were stimulated with phorbol-12-myristate-13-acetate $100 \mathrm{ng} /$ $\mathrm{mL}$ for $20 \mathrm{~min}$ at $37^{\circ} \mathrm{C}$. After washing with PBS-serum, $1 \mu \mathrm{L}$ of $2^{\prime}, 7^{\prime}$-dichlorofluorescin diacetate $5 \mu \mathrm{M}$ was added to detect reactive oxygen species (ROS) production. After a 15-min incubation period, fluorescence was read on a flow cytometer (FACS CANTO II, BD Biosciences, San Jose, CA, USA) and analysed using the FlowJo software (FlowJo, LLC).

\section{Statistical analysis}

All participants' baseline characteristics (-21d) were compared between groups using a General Linear Model. Mixed model analysis was used to test the effects of weight reduction (time), gluta- mine supplementation (treatment), and time by treatment interaction on all variables, except the symptoms of infection. Four different structures of covariance were tested to identify the best model for each data set according to the Schwarz's Bayesian criterion. Single-degree-of-freedom contrast analysis was used to test single effects. Kruskal-Wallis tests were conducted to compare the WURSS scores between the three groups at both $-1 \mathrm{~d}$ and $+5 \mathrm{~d}$ followed by the Conover post boc procedure. Frequencies (i.e., number of athletes reporting symptoms of infection and number of methods used to reduce weight) were compared between groups using the Fisher exact test. Data are described as mean \pm standard deviation and the level of significance was set at $5 \%$.

\section{RESULTS}

No significant differences in participants' characteristics were found between groups at baseline (body mass: $F=1.86, P=0.17$; body fat: $F=2.28 ; P=0.12$; age: $F=1.84 ; P=0.18$; height: $F=$ $0.74, P=0.49)$. The mean weight reduction, when combining both glutamine and placebo groups, was $5.7 \pm 2.3 \mathrm{~kg}(8.3 \% \pm$ $3.3 \%$ of body mass). There were no significant differences between groups for weight reduction (placebo: $6.1 \pm 2.4 \mathrm{~kg}$ or $8.5 \% \pm 2.4 \%$; glutamine: $5.2 \pm 2.1 \mathrm{~kg}$ or $8.2 \% \pm 4.1 \% ; P=0.86$; $95 \%$ confidence interval [CI], -2.67 to 3.18). Mixed models revealed a significant main effect of time for both absolute $(F=23.2$; $P<0.0001)$ and relative $(F=16.9, P<0.0001)$ body mass across the study, with no effect of group or group by time interaction being found $(P>0.05)$ (Fig. 2). Five days after competition, glutamine and placebo groups displayed a significant, yet incomplete, recovery of body mass (Fig. 2). In the control group, body mass remained stable (-21d: $86.1 \pm 25.9 \mathrm{~kg} ;-1 \mathrm{~d}: 86.8 \pm 26.4 \mathrm{~kg} ;+5 \mathrm{~d}$ : $87.1 \pm 25.8 \mathrm{~kg} ; F=0.01 ; P=0.99)$. Athletes in the glutamine and placebo groups used a combination of several weight loss methods (placebo: $2 \pm 1$ methods; glutamine: $2 \pm 1$ methods) which were intensified 3-5 days prior to competition and relied on severe energy restriction and dehydration (Supplementary Table 1).

The majority of athletes (74\%, or 17 out of 23 ) who reduced weight reported symptoms of upper respiratory infection at some point of the study, irrespective of group (glutamine group: 67\%, or 8 out of 12; placebo group: $72 \%$, or 8 out of $11, P=0.55$ ). In the control group, however, only 2 out of 8 athletes $(25 \%)$ reported symptoms of infection (control vs. glutamine: $P=0.08$; control vs. placebo: $P=0.05$ ). The Kruskal-Wallis test revealed significant differences for WURSS scores between groups at $-1 \mathrm{~d}$ $(P=0.019)$ but not at $+5 \mathrm{~d}(P=0.18)$. At $-1 \mathrm{~d}$, WURSS scores 
were not statistically different between glutamine and placebo groups $(P=0.13)$, highlighting the lack of effect of glutamine supplementation. On the other hand, both groups tended to report statistically higher scores than the control group (glutamine vs. control, $P=0.08$; placebo vs. control, $P=0.004$ ), highlighting the impact of rapid weight loss on the incidence of infection symptoms (Fig. 3).

Rapid weight loss led to significant changes in some parameters
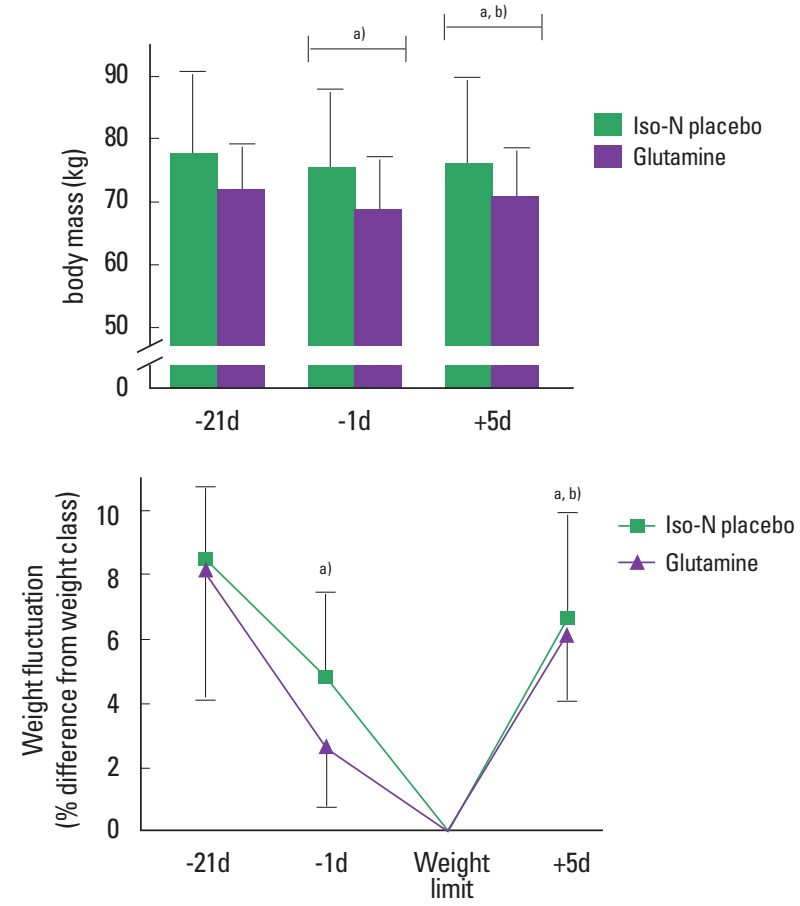

Fig. 2. Absolute (A) and relative (B) weight fluctuation throughout the study. Panel A depicts body mass relative to the individual weight class limit. Iso- $\mathrm{N}$, isonitrogenated placebo; $-21 d, 21$ days before; $-1 d, 1$ day before; $+5 d, 5$ days after. ${ }^{a}$ Different from -21d (main effect of time). ${ }^{b}$ Different from -1d (main effect of time). No significant between-group differences were observed. of immune cell function, but glutamine had no influence on these changes. Oxidative burst in monocytes and neutrophils remained unchanged throughout the study, regardless of supplementation (Table 1). In monocytes, we observed a minor fluctuation in
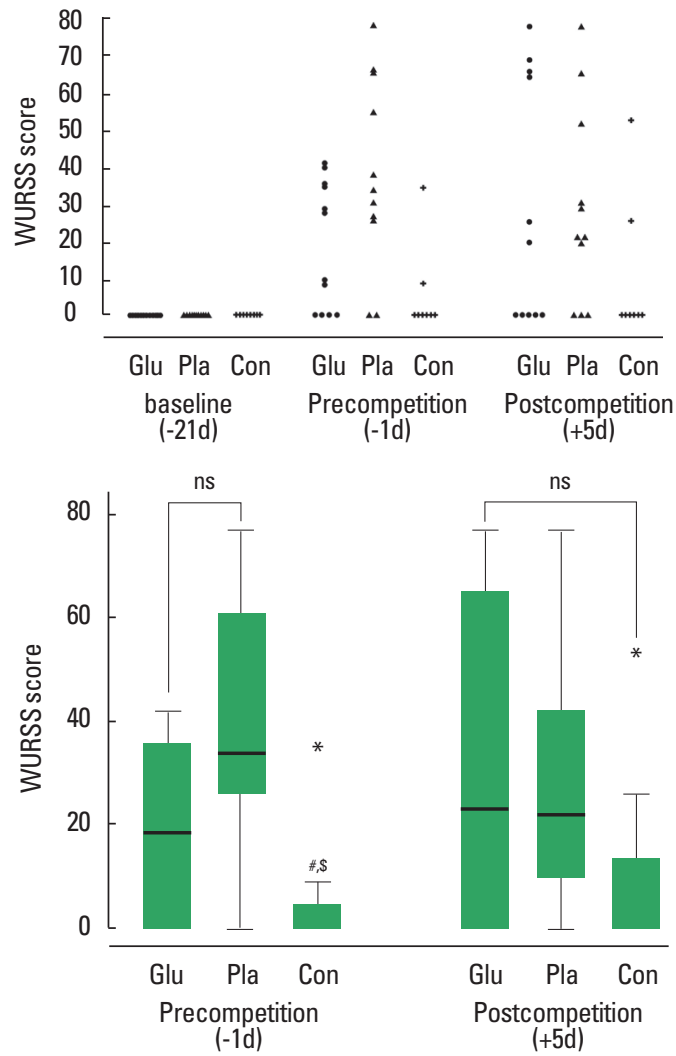

Fig. 3. (A) Individual Wisconsin upper respiratory symptom survey (WURSS) scores reported before and after weight loss in the glutamine (Glu), placebo (Pla), and control (Con) groups. (B) Median, interquartile intervals and range for WURSS scores in the glutamine, placebo and control groups 1 day before competition $(-1 \mathrm{~d})$ and 5 days following competition $(+5 \mathrm{~d})$ (asterisks denotes outliers). $-21 d, 21$ days before; $-1 d, 1$ day before; $+5 d, 5$ days after. Not significant (ns), $P>0.1 .{ }^{\#} P=0.08$ vs. glutamine. ${ }^{\$} P=0.004$ vs. placebo.

Table 1. Oxidative burst and phagocytic activity in monocytes and neutrophils before and after rapid weight loss

\begin{tabular}{|c|c|c|c|c|c|c|}
\hline \multirow{2}{*}{ Variable } & \multicolumn{3}{|c|}{ Iso-N placebo } & \multicolumn{3}{|c|}{ Glutamine } \\
\hline & $-21 d$ & $-1 d$ & $+5 d$ & $-21 d$ & $-1 d$ & $+5 d$ \\
\hline \multicolumn{7}{|c|}{ Oxidative burst (FI) } \\
\hline Monocyte & $1,418 \pm 1,284$ & $1,305 \pm 1,763$ & $1,164 \pm 708$ & $1,215 \pm 1,255$ & $2,381 \pm 1,854$ & $1,682 \pm 725$ \\
\hline Neutrophil & $8,016 \pm 9,594$ & $7,078 \pm 6,383$ & $7,012 \pm 5,948$ & $6,770 \pm 7,585$ & $7,734 \pm 5,021$ & $9,029 \pm 5,790$ \\
\hline \multicolumn{7}{|c|}{ Phagocytic activity (FI) } \\
\hline Monocyte & $4,421 \pm 3,634$ & $3,329 \pm 6,283^{a l}$ & $3,243 \pm 2,553$ & $4,051 \pm 3,186$ & $3,106 \pm 2,625^{b l}$ & $4,981 \pm 4,598^{c / 1}$ \\
\hline Neutrophil & $5,251 \pm 2,986$ & $17,428 \pm 22,374^{\mathrm{d} l}$ & $21,125 \pm 21,934^{e)}$ & $6,096 \pm 3,549$ & $11,029 \pm 17,113$ & $28,186 \pm 21,032$ \\
\hline
\end{tabular}

Values are presented as mean \pm standard deviation.

Iso-N, isonitrogenated placebo; -21d, 21 days before; -1d, 1 day before; $+5 \mathrm{~d}, 5$ days after; $\mathrm{Fl}$, fluorescence intensity; $\mathrm{ES}$, effect size.

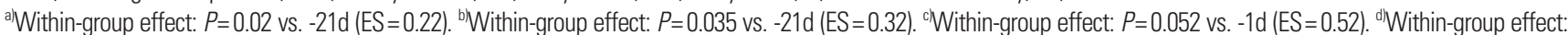
$P=0.058$ vs. $-21 \mathrm{~d}(\mathrm{ES}=0.96)$. ${ }^{\text {el }}$ Within-group effect: $P=0.048$ vs. $-21 \mathrm{~d}(\mathrm{ES}=1.27){ }^{f}$ Within-group effect: $P=0.009$ vs. $-21 \mathrm{~d}(\mathrm{ES}=1.80)$. 
phagocytic activity across the study (main effect of time: $F=5.5$, $P=0.01$ ) (Table 1). In neutrophils, the impact of rapid weight loss on phagocytic activity was more pronounced, with increased phagocytosis being observed in both groups at $-1 \mathrm{~d}$ and $+5 \mathrm{~d}$ in comparison to -21d (main effect of time: $F=7.4, P=0.004$ ) (Table 1). Glutamine supplementation did not influence phagocytic activity in monocytes (group by time interaction: $F=0.70, P=0.503$ ) or neutrophils (group by time interaction: $F=1.56, P=0.222$ ).

Rapid weight loss did not affect plasma glutamine concentrations (main effect of time: $F=1.24, P=0.27$ ) and glutamine supplementation was not effective in increasing plasma glutamine concentrations (group by time interaction: $F=1.21, P=0.32$ ) (Table 2). Serum cortisol was not influenced by weight loss (main effect of time: $F=1.23, P=0.79$ ) or supplementation (group by time interaction: $F=0.67, P=0.11$ ). Conversely, rapid weight loss significantly reduced total serum protein (main effect of time: $F=6.28, P=0.014$ ) and increased plasma $\mathrm{CK}$ levels (main effect of time: $F=3.31, P=0.019)$. Contrast analysis revealed that $\mathrm{CK}$ was elevated solely in the placebo group, remaining statistically similar to baseline in the glutamine group (Table 2).

Food intake analysis confirmed that athletes severely restricted food intake in the days preceding competition. Glutamine and

Table 2. Effects of rapid weight loss and glutamine supplementation on plasma glutamine, creatine kinase, serum cortisol, and total serum protein

\begin{tabular}{|c|c|c|c|}
\hline \multirow{2}{*}{ Group } & \multicolumn{3}{|c|}{ Time } \\
\hline & $-21 d$ & $-1 d$ & $+5 d$ \\
\hline \multicolumn{4}{|c|}{ Plasma glutamine $(\mu \mathrm{mol} / \mathrm{L})$} \\
\hline Placebo & $791 \pm 229$ & $749 \pm 230$ & $741 \pm 245$ \\
\hline Glutamine & $830 \pm 258$ & $835 \pm 243$ & $895 \pm 354$ \\
\hline Control & $1,075 \pm 161$ & $912 \pm 298$ & $1,004 \pm 392$ \\
\hline \multicolumn{4}{|c|}{ Total protein (mg/dL) } \\
\hline Placebo & $8.2 \pm 1.8$ & $6.9 \pm 1.6^{\text {a) }}$ & $7.4 \pm 2.0$ \\
\hline Glutamine & $8.3 \pm 1.7$ & $7.1 \pm 1.6^{b)}$ & $7.0 \pm 1.6$ \\
\hline Control & $7.2 \pm 1.5$ & $8.0 \pm 1.5$ & $6.9 \pm 1.3$ \\
\hline \multicolumn{4}{|c|}{ Plasma cortisol (ng/mL) } \\
\hline Placebo & $132 \pm 72$ & $118 \pm 57$ & $103 \pm 56$ \\
\hline Glutamine & $151 \pm 99$ & $121 \pm 71$ & $112 \pm 72$ \\
\hline Control & $142 \pm 125$ & $141 \pm 66$ & $154 \pm 75$ \\
\hline \multicolumn{4}{|c|}{ Creatine kinase (U/L) } \\
\hline Placebo & $125 \pm 54$ & $187 \pm 73.5^{c)}$ & $111 \pm 59^{d)}$ \\
\hline Glutamine & $136 \pm 58$ & $169 \pm 65$ & $130 \pm 64$ \\
\hline Control & $123 \pm 81$ & $116 \pm 45$ & $138 \pm 55$ \\
\hline
\end{tabular}

Values are presented as mean \pm standard deviation. $-21 d, 21$ days before; $-1 d, 1$ day before; $+5 d, 5$ days after.

a'Within-group effect: $P=0.0001$ vs. $-21 \mathrm{~d}(\mathrm{ES}=0.76)$. ${ }^{b}$ Within-group effect: $P=0.026$ vs. $-21 \mathrm{~d}\left(\mathrm{ES}=0.73\right.$ ). ${ }^{\mathrm{c}}$ Within-group effect: $P=0.02$ vs. $-21 \mathrm{~d}(\mathrm{ES}=0.92)$. ${ }^{\mathrm{d}}$ Within-group effect: $P=0.005$ vs. $-1 \mathrm{~d}(\mathrm{ES}=1.07)$.

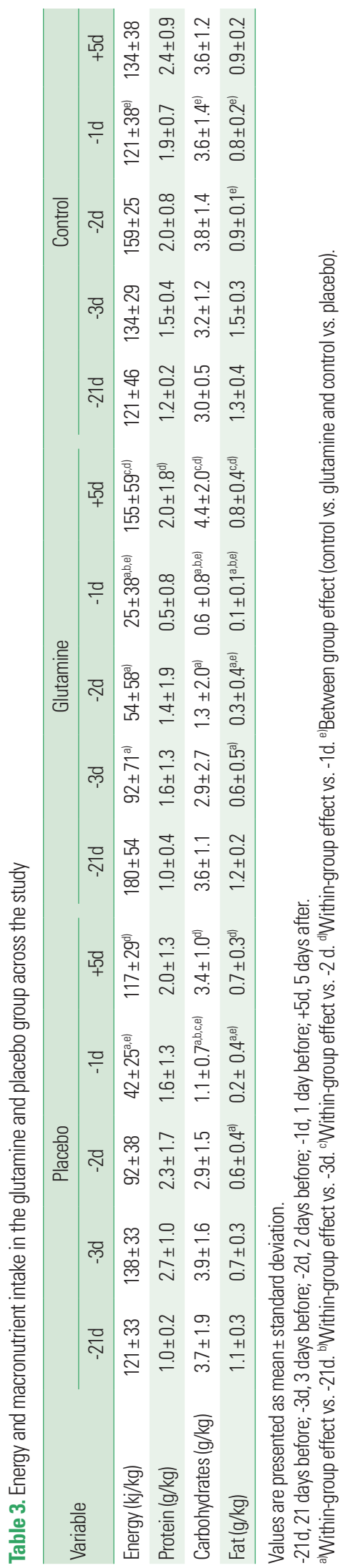


placebo groups significantly reduced energy and macronutrient intake in comparison to control group, but did not differ between each other (Table 3).

Five participants in the placebo group (42\%) and five in the glutamine group (45\%) were able to correctly identify the supplement they were taking. There was no difference between the correct supplement identification rate and the expected rate of correct identification from a random guess (Fischer exact test: $P=0.99$ ). Based on the number of returned leftover capsules, adherence to supplementation was $90 \% \pm 11 \%$ and $87 \% \pm 13 \%$ for glutamine and placebo groups, with no differences between groups being found (unpaired $t$-test: $P=0.41 ; 95 \% \mathrm{CI},-14.8$ to 6.3 ). Only two participants in the placebo group reported adverse gastrointestinal effects (stomach acid reflux and flatulence). No adverse effects were reported in the glutamine group.

\section{DISCUSSION}

The aggressive methods used by combat athletes to rapidly reduce their body weight include increased training loads and restricted food intake, both potentially capable of depleting circulating glutamine levels (Hankard et al., 1997; Walsh et al., 2000), and lead to impaired immune function and increased frequency of infections (Imai et al., 2002; Kowatari et al., 2001). Since critically low plasma glutamine has been implicated in immunosuppression (Castell et al., 1996; Curi et al., 1999), we hypothesized that immunosuppression in athletes undergoing rapid weight loss would be linked to reduced plasma glutamine and that glutamine supplementation could attenuate a potential immunosuppression by replenishing circulating glutamine levels. In our study, as expected, a clear increase in symptoms of infection was observed in both weight loss groups during and following rapid weight loss, but not in the control group. This suggests that athletes experienced some level of immunosuppression that was related to rapid weight loss. Surprisingly, rapid weight loss did not impact monocyte and neutrophil oxidative burst and an increase in neutrophil phagocytic activity was observed. Nevertheless, the increased frequency of infection symptoms was neither associated with plasma glutamine depletion, nor was glutamine supplementation capable of minimising them.

While exercise generally increases the phagocytic activity of neutrophil and monocytes, the negative effect of rapid weight loss on cell-mediated immunity highlights the role of restricted energy intake (Kowatari et al., 2001), increased exercise volume (Lakier Smith, 2003) and chronic hypohydration (Tsuchida et al.,
2004) on the immunosuppression accompanying rapid weight loss. In our study, we observed no effects of rapid weight loss on the neutrophil and monocyte ability to produce ROS, a fundamental microbicidal effector response. Similarly, we observed a statistically significant fluctuation in monocyte phagocytic activity, although the magnitude was rather small and likely of little physiological relevance. On the other hand, a considerably large increase in neutrophil phagocytic activity was observed, which could be interpreted as a lack of immunosuppressive effects of rapid weight loss; that is in opposition to the current literature. However, the improved neutrophil phagocytic activity could have been a mere reflex of decreased number of circulating neutrophils, a response that has been observed after intensive exercise and caloric restriction (Galassetti et al., 2006; Quindry et al., 2003). However, we did not measure neutrophil counts in this study. Our results concerning ROS and phagocytosis from circulating monocytes and neutrophils did not support to the notion that rapid weight loss is associated with decreased immunity. However, we did observe an increased frequency and severity of infection symptoms during and after weight reduction. Therefore, it is likely that other aspects of the immune response that we did not evaluate in this study, such as adaptive immune cells as well as humoral immunity and mucosal immunity, all of which have been implicated in rapid-weight-loss-associated immunosuppression (Font et al., 2002; Tsai et al., 2011), could have played a role in the increased susceptibility to opportunistic infections.

In contrast to our initial hypothesis, rapid weight loss did not lead to glutamine depletion. Thus, other factors unrelated to plasma glutamine concentrations likely contributed to the apparent immunosuppression. One explanation could relate to increased cortisol concentrations, which might affect some aspects of immune function (Pedersen and Hoffman-Goetz, 2000). However, studies have shown either minor increases in cortisol with values within the normal range (Degoutte et al., 2006) or no changes after rapid weight loss (Tsai et al., 2011). Therefore, the lack of an effect of rapid weight loss on cortisol shown here is in line with the literature and cannot explain the increased frequency of infection symptoms observed in our study. In fact, nutrient availability appears to have only a minor effect on cortisol levels (Ohta et al., 2002). Alternative explanations for the increased frequency of infection symptoms may relate to (a) intensified training loads, which have been associated with reduced circulating and mucosal levels of immunoglobulins (Gleeson et al., 1995; Svendsen et al., 2016), (b) reduced energy and/or macronutrient intake, which has also been linked to impairments in several aspects of the cellular, 
systemic and mucosal immune defenses (Papacosta and Gleeson, 2013), or (c) a combination of both.

Previous reports have suggested that the lack of positive effects of glutamine supplementation in humans could be explained by the fact that plasma glutamine depletion must be severe to limit immune function. Despite the severe energy restriction and increased training loads in the days preceding competition, none of the athletes showed plasma glutamine levels below $400 \mathrm{mmol} / \mathrm{L}$. Thus, the lack of effect of glutamine supplementation on symptoms of infection can be explained by the remarkably stable plasma glutamine across the study, despite the extreme weight fluctuation and the aggressive methods used to reduce weight. Interestingly, plasma glutamine levels were not elevated in the glutamine group. This, however, is likely explained by the kinetics of appearance and removal of glutamine in blood after oral ingestion (Venkatraman and Pendergast, 2002). The single doses used in the present investigation $(7 \mathrm{~g})$ are similar to that previously shown to elevate plasma glutamine (Venkatraman and Pendergast, 2002). Since our supplementation protocol required athletes to ingest $7 \mathrm{~g}$ every $4-5 \mathrm{hr}$, it is reasonable to assume that plasma glutamine levels were constantly elevated in the glutamine group (Venkatraman and Pendergast, 2002).

In the present investigation, plasma CK was increased after rapid weight loss in the placebo group only, with a nonsignificant increase being observed in the glutamine group and no change in the control group. These results were somewhat expected, since previous studies have shown that rapid weight loss induces a significant alteration in CK levels (Ziegler et al., 1990). This suggests that glutamine might play a role in attenuating muscle damage after exhaustive exercise, which is in accordance with previous research (Umeda et al., 2004). However, all plasma CK values were within the normal range across the study. Therefore, our data does not support any clinical benefit of glutamine in attenuating muscle damage.

The present study is not without its limitations. First, we were unable to access athletes for blood collection on competition day (i.e., when weight loss peaks. Second, we were unable to perform a comprehensive examination of the immune function. Finally, we acknowledge the limitations of self-reported infection symptoms, as they could be related to noninfectious causes, such as allergy and mucosal inflammation. However, it is very unlikely that allergy and non-infectious inflammation could explain the increase in symptoms frequency only in the groups reducing weight but not in controls.

To conclude, the present study confirmed that rapid weight loss leads to increased frequency of symptoms of infection. However, this was not linked to decreased glutamine levels, nor was glutamine supplementation capable of counteracting the apparent decreased immunity during rapid weight loss. Immunosuppression induced by rapid weight loss is not related to circulating glutamine levels; therefore, glutamine supplementation is not beneficial for supporting immune function in combat athletes undergoing rapid weight loss.

\section{CONFLICT OF INTEREST}

No potential conflict of interest relevant to this article was reported.

\section{ACKNOWLEDGMENTS}

This research was supported by Sao Paulo Research Foundation (FAPESP grant \#11/22845-7) and National Council for Scientific and Technological Development (CNPq). The authors are thankful to Senseis Hatiro and Garcia for facilitating contact with the athletes as well as Meire I Hiyane, Dr Ana Lúcia de Sá Pinto, Dra Fabiana Benatti, Dra Nathália Figueirêdo and Dr Antonio H Lancha Jr for their technical contributions. Finally, we are grateful to Dr Bryan Saunders and Eimear Dolan for carefully proofreading this manuscript.

\section{SUPPLEMENTARY MATERIAL}

Supplementary Table 1 can be found via https://doi.org/10.129 65/jer.1835154.577.

\section{REFERENCES}

Ardawi MS, Newsholme EA. Glutamine metabolism in lymphocytes of the rat. Biochem J 1983;212:835-842.

Artioli GG, Gualano B, Franchini E, Scagliusi FB, Takesian M, Fuchs M, Lancha AH Jr. Prevalence, magnitude, and methods of rapid weight loss among judo competitors. Med Sci Sports Exerc 2010;42:436-442.

Artioli GG, Saunders B, Iglesias RT, Franchini E. It is Time to Ban Rapid Weight Loss from Combat Sports. Sports Med 2016;46:1579-1584.

Blanchard MA, Jordan G, Desbrow B, MacKinnon LT, Jenkins DG. The influence of diet and exercise on muscle and plasma glutamine concentrations. Med Sci Sports Exerc 2001;33:69-74.

Castell LM, Poortmans JR, Newsholme EA. Does glutamine have a role in reducing infections in athletes? Eur J Appl Physiol Occup Physiol 
1996;73:488-490.

Curi R, Newsholme P, Pithon-Curi TC, Pires-de-Melo M, Garcia C, Homem-de-Bittencourt Júnior PI, Guimarães AR. Metabolic fate of glutamine in lymphocytes, macrophages and neutrophils. Braz J Med Biol Res 1999;32:15-21.

Cury-Boaventura MF, Levada-Pires AC, Folador A, Gorjão R, Alba-Loureiro TC, Hirabara SM, Peres FP, Silva PR, Curi R, Pithon-Curi TC. Effects of exercise on leukocyte death: prevention by hydrolyzed whey protein enriched with glutamine dipeptide. Eur J Appl Physiol 2008; 103:289-294.

Degoutte F, Jouanel P, Bègue RJ, Colombier M, Lac G, Pequignot JM, Filaire E. Food restriction, performance, biochemical, psychological, and endocrine changes in judo athletes. Int J Sports Med 2006;27:9-18.

Font J, Ramos-Casals M, Vilas AP, García-Carrasco M, Brito MP, de la Red G, Gil V, García-Carrasco A, Cervera R, Ingelmo M. Low values of creatine kinase in systemic lupus erythematosus. Clinical significance in 300 patients. Clin Exp Rheumatol 2002;20:837-840.

Galassetti PR, Nemet D, Pescatello A, Rose-Gottron C, Larson J, Cooper DM. Exercise, caloric restriction, and systemic oxidative stress. J Investig Med 2006;54:67-75.

Gleeson M. Dosing and efficacy of glutamine supplementation in human exercise and sport training. J Nutr 2008;138:2045S-2049S.

Gleeson M, McDonald WA, Cripps AW, Pyne DB, Clancy RL, Fricker PA. The effect on immunity of long-term intensive training in elite swimmers. Clin Exp Immunol 1995;102:210-216.

Hankard RG, Haymond MW, Darmaun D. Role of glutamine as a glucose precursor in fasting humans. Diabetes 1997;46:1535-1541.

Housh TJ, Johnson GO, Kenney KB, McDowell SL, Hughes RA, Cisar CJ, Thorland WG. Validity of anthropometric estimations of body composition in high school wrestlers. Res Q Exerc Sport 1989;60:239-245.

Imai T, Seki S, Dobashi H, Ohkawa T, Habu Y, Hiraide H. Effect of weight loss on T-cell receptor-mediated T-cell function in elite athletes. Med Sci Sports Exerc 2002;34:245-250.

Keast D, Arstein D, Harper W, Fry RW, Morton AR. Depression of plasma glutamine concentration after exercise stress and its possible influence on the immune system. Med J Aust 1995;162:15-18.

Kingsbury KJ, Kay L, Hjelm M. Contrasting plasma free amino acid patterns in elite athletes: association with fatigue and infection. Br J Sports Med 1998;32:25-32.

Kowatari K, Umeda T, Shimoyama T, Nakaji S, Yamamoto Y, Sugawara K. Exercise training and energy restriction decrease neutrophil phagocytic activity in judoists. Med Sci Sports Exerc 2001;33:519-524.

Krzywkowski K, Petersen EW, Ostrowski K, Link-Amster H, Boza J, Halkjaer-Kristensen J, Pedersen BK. Effect of glutamine and protein supplementation on exercise-induced decreases in salivary IgA. J
Appl Physiol (1985) 2001a;91:832-838.

Krzywkowski K, Petersen EW, Ostrowski K, Kristensen JH, Boza J, Pedersen BK. Effect of glutamine supplementation on exercise-induced changes in lymphocyte function. Am J Physiol Cell Physiol 2001b;281: C1259-C1265.

Lagranha CJ, Alba-Loureiro TC, Martins EF, Pithon-Curi TC, Curi R. Neutrophil fatty acid composition: effect of a single session of exercise and glutamine supplementation. Amino Acids 2008;35:243-245.

Lagranha CJ, de Lima TM, Senna SM, Doi SQ, Curi R, Pithon-Curi TC. The effect of glutamine supplementation on the function of neutrophils from exercised rats. Cell Biochem Funct 2005;23:101-107.

Lakier Smith L. Overtraining, excessive exercise, and altered immunity: is this a T helper-1 versus T helper-2 lymphocyte response? Sports Med 2003;33:347-364.

Novak F, Heyland DK, Avenell A, Drover JW, Su X. Glutamine supplementation in serious illness: a systematic review of the evidence. Crit Care Med 2002;30:2022-2029.

Ohta S, Nakaji S, Suzuki K, Totsuka M, Umeda T, Sugawara K. Depressed humoral immunity after weight reduction in competitive judoists. Luminescence 2002;17:150-157.

Papacosta E, Gleeson M. Effects of intensified training and taper on immune function. Rev Bras Educ Fis Esporte 2013;27:159-176.

Parry-Billings M, Evans J, Calder PC, Newsholme EA. Does glutamine contribute to immunosuppression after major burns? Lancet 1990;336: 523-525.

Pedersen BK, Hoffman-Goetz L. Exercise and the immune system: regulation, integration, and adaptation. Physiol Rev 2000;80:1055-1081.

Quindry JC, Stone WL, King J, Broeder CE. The effects of acute exercise on neutrophils and plasma oxidative stress. Med Sci Sports Exerc 2003;35:1139-1145.

Sasaki E, Umeda T, Takahashi I, Arata K, Yamamoto Y, Tanabe M, Oyamada K, Hashizume E, Nakaji S. Effect of glutamine supplementation on neutrophil function in male judoists. Luminescence 2013;28:442449.

Svendsen IS, Killer SC, Carter JM, Randell RK, Jeukendrup AE, Gleeson $\mathrm{M}$. Impact of intensified training and carbohydrate supplementation on immunity and markers of overreaching in highly trained cyclists. Eur J Appl Physiol 2016;116:867-877.

Tsai ML, Chou KM, Chang CK, Fang SH. Changes of mucosal immunity and antioxidation activity in elite male Taiwanese taekwondo athletes associated with intensive training and rapid weight loss. Br J Sports Med 2011;45:729-734.

Tsuchida M, Nagura T, Bai XF, Li CC, Tomiyama-Miyaji C, Kawamura T, Uchiyama M, Abo T. Granulocytic activation and reciprocal immunosuppression induced by dehydration: relationship with renal failure. 
Biomed Res 2004;25:171-178.

Umeda T, Nakaji S, Shimoyama T, Yamamoto Y, Totsuka M, Sugawara K. Adverse effects of energy restriction on myogenic enzymes in judoists. J Sports Sci 2004;22:329-338.

Venkatraman JT, Pendergast DR. Effect of dietary intake on immune function in athletes. Sports Med 2002;32:323-337.

Walsh NP, Blannin AK, Bishop NC, Robson PJ, Gleeson M. Effect of oral glutamine supplementation on human neutrophil lipopolysaccharide-stimulated degranulation following prolonged exercise. Int J Sport Nutr Exerc Metab 2000;10:39-50.

Ziegler TR, Benfell K, Smith RJ, Young LS, Brown E, Ferrari-Baliviera E, Lowe DK, Wilmore DW. Safety and metabolic effects of L-glutamine administration in humans. JPEN J Parenter Enteral Nutr 1990;14(4 Suppl):137S-146S. 